\title{
Stress level assessment among health care workers involved in the management of critically ill COVID-19 patients
}

\author{
Tharwat Aisa' ${ }^{1}$ (D) Dara Diviney ${ }^{1}$. Jubil Thomas ${ }^{1} \cdot$ Nada Al Qadheeb $^{2} \cdot$ Moamen Abdelbaky $^{3} \cdot$ Hosam Afify $^{3}$. \\ Mustafa Yasawy ${ }^{4} \cdot$ Mohamad Mahmoud $^{5} \cdot$ Ahmed Abdallah $^{6} \cdot$ Asma Bashir $^{7} \cdot$ Khaled Algrni $^{8} \cdot$ Basheer Elgammal $^{9}$
}

Received: 17 February 2021 / Accepted: 17 July 2021 / Published online: 31 July 2021

(c) Royal Academy of Medicine in Ireland 2021

\begin{abstract}
Background Intensive care health care workers (HCWs) are frontlines of this crisis as they deal with critically ill COVID-19 patients which can potentially affect their mental well-being and causes different levels of stress.

Aim To determine the prevalence of stress among HCWs involved in the management of critically ill COVID-19 patient, identify the factors associated with stress, and highlight the availability of psychological support provided to HCWs.

Methods A cross-sectional multicenter, international study using a web-based questionnaire of 27 questions including the Perceived Stress Scale-10 (PSS-10) for assessment of stress level. Questions to identify factors associated with stress, the psychological support provided, and the sociodemographic characteristics were included.

Results We received a total 1649 responses from 59 countries: 550 (34\%) were from Europe, 525 (32.36\%) from Asia, 283 (17.44\%) from Africa, 177 (11\%) from America, and 88 (5.42\%) from Australia. The average stress level was 22 points on the PSS denoting moderate stress in 1327 (81.8\%) respondents, while 239 (14.73\%) respondents had a severe level of stress. Female gender, working in high capacity units and remote areas in addition to lack of psychological support, was significantly associated with stress in our study.

Conclusion Stress level was moderate to severe among intensive care HCWs during this pandemic, and many factors were associated with stress emphasizing the importance of psychological support during that unprecedented pandemic.
\end{abstract}

Keywords COVID-19 $\cdot$ Health care workers $\cdot$ Intensive care $\cdot$ Stress level

\section{Introduction}

Coronaviruses are important human and animal pathogens. In December 2019, a novel coronavirus was identified as the cause of a cluster of pneumonia cases in Wuhan, a city in the Hubei Province of China. It rapidly spread, resulting in an epidemic throughout China, followed by an increasing

Tharwat Aisa

Tharwat40@yahoo.com

$1 \quad$ Anesthesia and Adult Intensive Care Department, RCSI Louth Hospitals, Our Lady of Lourdes Hospital, Drogheda, Ireland

2 Human Research Protection Administration, King Khalid Medical City, Research Center Dhahran, Saudi Arabia

3 Adult Intensive Care Department, King Abdullah Medical City, Makkah, Saudi Arabia

4 Adult Critical Care Department, King Fahad Medical City, Riyadh, Saudi Arabia number of cases in other countries throughout the world. In February 2020, the World Health Organization designated "COVID-19" pandemic, as an ongoing pandemic for coronavirus disease 2019 [1]. The virus that causes COVID-19 is named severe acute respiratory syndrome coronavirus 2 (SARS-CoV-2); previously, it was referred to as 2019$\mathrm{nCoV}$. Therefore, the declaration of the Novel Coronavirus

5 Anesthesia and Adult Intensive Care Department, Manchester Royal Infirmary, Manchester, UK

6 Critical Care Department, Hunter New England Health District, Newcastle, Australia

7 Institute of Pharmaceutical Sciences, Jinnah Sindh Medical University, Karachi, Pakistan

8 College of Public Health and Health Informatics, Umm Al-Qura University, Makkah, Saudi Arabia

9 Anesthesia and Critical Care Department, Mater University Hospital, Dublin, Ireland 
(COVID-19) as a pandemic leads to the implementation of surge plans and an increase in the number of intensive care unit (ICU) beds across most health care facilities. The ICU can be a stressful environment for patients, relatives, and health care professionals. Growing evidence suggests that stress and burnout among ICU nurses [2] and ICU physicians is the result of the demanding and continuously high stressful work environment [3, 4]. Additionally, health care workers (HCWs) in the ICU are frontlines of this crisis as they deal with critically ill patients affected by COVID-19 which can potentially affect their mental well-being and causes different levels of stress and psychological disorders.

The Perceived Stress Scale-10 (PSS-10) was developed to measure the degree to which situations in one's life are appraised as stressful. The PSS-10 was published in 1983 [5] and has become one of the most widely used tools with a satisfactory reliability [6]. The tool is composed of 10 items and was found to correlate well with other stress measures, self-reported health and health service measures, health behavior measures, smoking status, and help-seeking behavior [5]. The perceived stress scale categorizes participants into three groups based on their score. Participants who score 0-13 are considered low stress, while participants who score 14-26 are considered moderate stress, and finally, participants who score $27-40$ are considered high stress. In this study, we sought to determine the prevalence of stress among HCWs involved in the management of critically ill COVID-19 patients, identify the factors associated with stress, and highlight the availability of psychological support provided to HCWs.

\section{Methods}

This is a cross-sectional multicenter, international study. A web-based survey was conducted in order to collect the responses from health care workers, working in the ICU settings and are in direct involvement with the management of COVID-19 patients. Participation was voluntary and anonymous (no personal data collected). All the participants gave their consent for participating in the study by initial approval in the front page of the survey. This study received institutional review board (IRB) approval from King Fahad Medical City Research Centre-Riyadh number 20-294 and from King Abdullah Medical City Research Center with IRB number 20-637, Kingdom of Saudi Arabia. The survey targeted all the HCWs who were involved in the management of critically ill COVID-19 patients. The design of the survey consisted of 27 questions divided into three parts, the first part covered participants work experience, sociodemographic data, and institutional work characteristics which included the age, gender, staff category, type of the hospital, locations, and ICU occupancy which sub-categorized into below the usual ICU capacity, the usual capacity, above capacity (means that the ICU was fully occupied with patients and extending to the high dependency unit/beds in the hospital), and well above capacity (means that the ICU was fully occupied with patients and extending to other areas in the hospital like the operating theatres, recovery areas, or the wards as surge plans). The second part included information surrounding PSS-10 to measure the stress level. In the third part, we collected the factors associated with stress in addition to the categories of psychological support provided for the HCWs during the pandemic. We presumed that the hospitals initiated campaigns and teams for the psychological support of their staff during the pandemic, which include psychiatric counselling, phone calls, or other ways of communications to actively listen and manage the HCWs' concerns (Supplementary Appendix A).

The questions were developed and reviewed by the steering committee and a pilot test was performed to test the ease, flow, and the time spent to do the survey. The final version was then revised and designed on the Surveymonkey ${ }^{\circledR}$ online platform (SVMK Inc., San Mateo, USA) and posted at https://www. surveymonkey.com/r/3MNSV5N. Only the English language version was available and we reported data collected between 24/04/2020 and 13/07/2020. We invited HCWs to participate through steering committee members, social medias, and personal networks of the management committee members.

\section{Data analysis}

All the survey results were exported and analyzed using SPSS (Statistical Package for the Social Sciences) for windows version 18.00 (SPSS Inc., Chicago, IL, USA) program, and ANOVA with Tukey's multiple comparison was used to examine the differences in the stress level among the groups. $P$-value was considered significant if it was less than 0.05 . Because some variables showed missing values which have been contributed to lower sample size in some analysis, ultimately, the total number for analysis has been dropped to 1623 . Multiple logistic regression was used to examine the significant predictors that contribute to the high level of stress and a model to examine the different kinds of psychological support as predictor of low stress level. A stepwise predictor selection was used to select the most significant predictors. Despite the fact that some variables might be statistically significant if examined individually, however, they were not clinically significant when being examined without taking into account the other factors.

\section{Results}

We received a total 1649 responses from 59 countries. Because some variables showed missing values which might have been contributed to lower total number in the 
sample size in some analysis, the total responses were 557 (34\%) from Europe, 531 (32.36\%) from Asia, 284 (17.44\%) from Africa, 189 (11\%) from America, and 88 (5.42\%) from Australia. A total of 690 (43.95\%) were senior doctors, 491 (30.43\%) were nurses, 302 (18.60\%) were junior doctors, and 133 (7.04\%) others (clinical pharmacists, physiotherapists, and respiratory therapists). Responders were mainly male 888 (54.81\%). The overall average stress level was 22 points on the PSS-10 denoting moderate stress in 1327 $(81.8 \%)$ respondents, while $239(14.73 \%)$ respondents had a severe level of stress, and only $56(3.45 \%)$ had mild stress.

\section{Mean stress level in the participants}

The mean stress level in the participants according to the sociodemographic characteristics with Tukey's multiple comparisons. The young HCWs (18-34 years) had the highest stress level compared to all other age groups where participants whose ages $\geq 55$ years had significantly lower stress than the other groups. The stress level among females was significantly higher than males.

Looking at the different categories, nurses had an average score of 22.95 on the PSS-10 which was significantly higher than all the other groups. Furthermore, a strong association was determined between the ICU bed occupancy and the level of stress. Participants who reported that bed occupancy of their hospital was above capacity or well above capacity had a higher stress level compared to others $(22.82,23.25$, respectively). Finally, HCWs who worked in Europe had stress level that is significantly higher than all the other continents (Table 1).

\section{Predictors of high stress}

Model 1 predicts high stress level based on the significantly correlated variables (gender, ICU current occupancy, and type of the hospital and continent). The overall model was
Table 1 The mean Perceived Stress Scale by sociodemographic characteristics

\begin{tabular}{|c|c|c|c|c|}
\hline & $\mathrm{M}$ & $\mathrm{SD}$ & $\mathrm{F}$ & Multiple comparisons \\
\hline \multicolumn{5}{|l|}{ Age } \\
\hline $1.18-34$ & 22.61 & 4.9 & \multirow{4}{*}{$9.75 * * *$} & $1>2,3,4 * * *$ \\
\hline $2.35-44$ & 21.88 & 5.3 & & $2>4 * * *$ \\
\hline $3.45-54$ & 21.42 & 4.5 & & $3>4 * * *$ \\
\hline $4.55+$ & 19.20 & 5.7 & & \\
\hline \multicolumn{5}{|l|}{ Gender } \\
\hline 1. Male & 21.33 & 5.2 & & \multirow[t]{2}{*}{$2>1 * * *$} \\
\hline 2. Female & 22.87 & 4.8 & $37.4 * * *$ & \\
\hline \multicolumn{5}{|l|}{ Category } \\
\hline 1. Senior doctor & 21.61 & 5.4 & \multirow{4}{*}{$7.70 * * *$} & \multirow{4}{*}{$3>1,2,4 * * *$} \\
\hline 2. Doctor, SHO trainee, registrar & 21.71 & 4.5 & & \\
\hline 3. Nurse & 22.95 & 5.2 & & \\
\hline 4. Others & 21.54 & 4.1 & & \\
\hline \multicolumn{5}{|l|}{ ICU current occupancy } \\
\hline 1. Below capacity & 20.27 & 4.9 & \multirow{4}{*}{$30.8 * * *$} & \\
\hline 2. Usual capacity & 20.42 & 4.6 & & $3>1,2 * * *$ \\
\hline 3. Above capacity & 22.82 & 5.2 & & $4>1,2 * * *$ \\
\hline 4. Well above capacity & 23.25 & 4.3 & & \\
\hline \multicolumn{5}{|l|}{ Type of hospital } \\
\hline 1. Local/community hospital & 21.29 & 3.9 & \multirow[t]{4}{*}{$5.16 * *$} & \multirow[t]{4}{*}{$2>1,3 * * *$} \\
\hline 2. Large urban/regional teaching hospital & 22.73 & 5.5 & & \\
\hline 3. Large tertiary/quaternary care/teaching & 21.90 & 5.3 & & \\
\hline 4. Other (incl. private) & 21.89 & 4.8 & & \\
\hline \multicolumn{5}{|l|}{ Continent } \\
\hline 1. Europe & 23.66 & 5.2 & \multirow[t]{5}{*}{$23.6^{* * *}$} & \multirow[t]{5}{*}{$1>2,3,4,5^{* * *}$} \\
\hline 2. Asia & 21.49 & 5.7 & & \\
\hline 3. Australia & 20.28 & 4.9 & & \\
\hline 4. Americans & 20.93 & 3.5 & & \\
\hline 5. Africa & 21.10 & 3.6 & & \\
\hline
\end{tabular}

$F$ values correspond to the ANOVA test for difference in means

$* p<0.10, * * p<0.05, * * * p<0.01$

Multiple comparisons were done using Tukey's method

$<$ and $>$ indicate significant difference between the numbered groups according to Tukey's multiple comparisons method 
significant $(p<0.0001)$. The odds of female having high stress level are $1.88(1.43-2.45)$ times the odds of high stress among males while controlling the ICU occupancy and type of the hospital $(p<0.001)$. The odds of having high stress level among participants whom current ICU occupancy was well above occupancy are 4.98 (2.81-8.24) times those who reported ICU below capacity while controlling gender and type of the hospital and continent $(p<0.001)$. The odds of having high stress level among participants whom current ICU occupancy was above occupancy are 3.33 (2.12-5.70) times those who reported ICU below capacity while controlling gender and type of the hospital $(p<0.001)$. Participants who reported that they worked in other types of hospitals (including the private sector) had higher odds of having high stress level compared to local/community hospitals $(\mathrm{OR}=2.15(1.22-3.80)$ and $p$-value $<0.001)$. Participants from the continent of Americas or Africa have shown less high stress level than European peers $(\mathrm{OR}=0.56$ (0.38-0.83), 0.32 (0.19-0.54), respectively). The participants from European countries showed higher level of stress than those from African/American countries $(\mathrm{OR}=0.56$ (0.38-0.83), 0.32 (0.19-0.54)) (Table 2).

\section{Types of psychological support}

In model 2, we are modeling for low stress level, the participants who receive psychiatric team counselling have 1.92 (1.35-2.71) the odds of having low stress level compared to those who did not receive psychiatric team consoling while

Table 2 Multiple logistic regression for high stress (model 1)

\begin{tabular}{ll}
\hline Predictor & $\begin{array}{l}\text { Model 1, }(n=1594) \\
\text { AOR }(95 \% \mathrm{CI})\end{array}$ \\
\hline Gender & $1.88(1.43-2.45)^{* * * *}$ \\
Female & 1.00 \\
Male & \\
ICU current occupancy & $4.98(2.81-8.24)^{* * * *}$ \\
Well above capacity & $3.33(2.12-5.70)^{* * *}$ \\
Above capacity & $1.55(0.91-2.64)$ \\
Usual capacity & 1.00 \\
Below capacity & \\
Type of hospital & $2.15(1.22-3.80)^{* * *}$ \\
Other (remote \& private) & $0.93(0.62-1.39)$ \\
Large tertiary/quaternary care/teaching & $1.06(0.69-1.61)$ \\
Large urban/regional teaching hospital & 1.00 \\
Local/community hospital & \\
Continent & $0.56(0.38-0.83)^{* * *}$ \\
Africa & $0.32(0.19-0.54)^{* * *}$ \\
Americas & $0.77(0.54-1.09)$ \\
Asia & $0.50(0.25-1.02)$ \\
Australia & 1.0 \\
Europe &
\end{tabular}

$n$, number; $A O R$, adjusted odds ratio; $C I$, confidence interval controlling other psychological support types. The odds of having low stress for those who receive motivational and positive emails and messages are 1.84 (1.34-2.52) times the odds for those who did not receive such kind of support while controlling other psychological support types (Table 3).

\section{Fears and concerns}

Fear of the infection was the major concern for more than $80 \%$ of all the respondents. A total of $949(58.5 \%)$ reported that fear of getting infected with COVID-19 increased their stress level while 384 (23.67\%) reported that the fear of transmitting the infection to their families was the main cause of their stress. Other contributing factors to stress were the increased duty hours, the increased work load, and shortage of staff as reported by $118(7.27 \%)$ while the lack of (personal protective equipments) PPEs accounted for stress in 91 (5.61\%) respondents. Social media and triaging COVID patients were the least causes of staff stress as quoted by 50 (3\%) and 49 (3\%), respectively (Fig. 1).

A total of $979(60.17 \%)$ of the respondents mentioned that increasing the number of the staff is importantly required in order to alleviate their stress level while 902 (55.44\%) asked for decreasing the duty hours as an action to decrease their stress. Moreover, 726 (44.62\%) asked for psychiatric team counselling to help manage their stress during the pandemic.

\section{Discussion}

Our results describe the prevalence of stress levels of HCWs involved in the management of critically ill COVID-19 patients. This pandemic is unique in its rapid transmission and it results in not only public health concerns, but also it

Table 3 Multiple logistic regression for low stress based on types of psychological support received (model 2)

\begin{tabular}{ll}
\hline Predictor & $\begin{array}{l}\text { Model 2, }(n=1594) \\
\text { AOR }(95 \% \text { CI })\end{array}$ \\
\hline $\begin{array}{l}\text { Psychiatric team counselling } \\
\text { Yes }(27.34 \%)\end{array}$ & $1.92(1.35-2.71)^{* * *}$ \\
No (72.66) & 1.00 \\
Phone calls & \\
Yes (45.09\%) & $1.15(0.88-1.52)$ \\
No (54.91\%) & 1.00 \\
Motivational and positive emails, messages & \\
Yes $(53.43 \%)$ & $1.84(1.34-2.52)^{* * *}$ \\
No (46.57\%) & 1.00 \\
Active listening to your concerns & \\
Yes (38.32\%) & $0.99(0.72-1.37)$ \\
No $(61.68 \%)$ & 1.00 \\
\hline
\end{tabular}




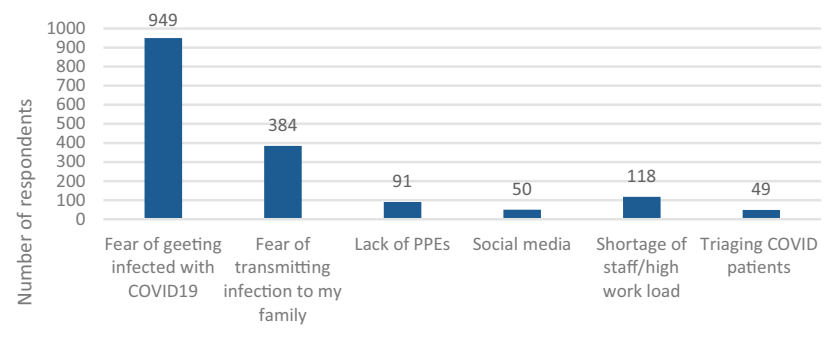

Fig. 1 Contributing factors for stress as per respondents

causes tremendous stress for frontline HCWs. Overall, the sample showed a PSS-10 score of 22 which is higher than that reported in the general population $<13$ according to Cohen and Williamson [7] and close to the values reported by Chua et al. [8], during the SARS outbreak in 2003, which placed it at 18.5 .

In our cohort, the prevalence of high stress level was $14.73 \%$, while the prevalence of moderate level of stress was $81.8 \%$. As already pointed out, some studies done in the past reported that HCWs, especially those working in emergency units, intensive care units, and infectious disease wards, are at higher risk of developing adverse psychiatric impact [9]. In general, HCWs are at the first line of the battle under the influence of high risk of getting infected and the high risk of mortality from COVID-19.

In addition to the intense media coverage with reports from different countries which address a high rate of infections in the health care professionals, they will undoubtedly show the emotion of fear, anxiety, anger, and frustration [10, 11].

Zhu et al. [12] from Wuhan, China, the epicenter of the virus outbreak, have reported that among 5,062 HCPs, the prevalence rate of stress was $29.8 \%$, while another study from China by Lai et al. [13] reported that the prevalence rate of severe distress was $10.5 \%$ among 1,257 HCWs.

We found that the females were more significantly stressed than the males (OR: $1.88 P=0.001)$. This finding is in line with the findings reported by Lai et al. [13], where women are at increased odds of developing distress (OR: $1.45 ; P=0.01)$.

Similar results were described by the initial author Cohen and Janicki-Deverts [14] and Remor [15], regarding PSS and PSS-10, women received significantly higher perceived stress scores than men. The reasons for this finding may be related to sex differences in coping with stress. The findings in this paper point out higher levels of anxiety and worry in women. Numerous studies, Uhlenhuth and Paykel [16], show how women report higher intensity of the symptoms than men, and Kendler et al. [17] display gender and sensitivity to the depressogenic effect of stressful life events, where women reported higher stress rates. The study of Dalgard et al. [18] explains why with a more affiliative style, and a stronger involvement in household and family matters, women are more exposed to problems in the social network. Based on this, women are more likely than men to report events in the social network.

Nurses showed higher level of stress than the other specialties (18.94\%); meanwhile, junior doctors reported higher stress levels than senior doctors. That was reported in some other studies such as that by Rossi et al. [19] in Italy where the younger age and female sex were associated with higher levels of stress, anxiety, and depression during the pandemic and nurses suffered more from insomnia. Frontline nurses treating patients with COVID-19 are likely exposed to the highest risk of infection because of their close, frequent contact with patients and working longer hours than usual $[20,21]$. During the SARS outbreak, a study conducted among health care workers in emergency departments also showed that nurses were more likely to develop distress and use behavioral disengagement than physicians [22]. They were physically and psychologically challenged when committing themselves to providing high-quality nursing care for patients [21, 23-25]. Moreover, at the early stage of the SARS epidemic, nurses may have been less likely to be warned about exposure or provided with adequate protections [25]. Therefore, it warrants a particular attention to the mental health well-being of the nurses and female HCWs during the COVID-19 pandemic. The higher stress level in junior doctors was reported in many previous studies which highlighted many potential sources for their stress. It could be explained by greater work load, long working hours, less experiences, and high job demands. Moreover, junior doctors might feel insecurity regarding career progression and work-life imbalance [26-29]. We found, however, the HCWs who worked in the units with surge beds above capacity had higher levels of stress which is logically explained by the overall excessive work load.

The respondents from European countries were more stressed than those from the other countries (African, American, and Asian countries) which could be related to the surge of the cases during the early beginning of its spread throughout European countries with unpreparedness of the health care facilities to deal with the pandemic in some areas and shortage of the PPEs in some hospitals during the surge time. The expectations of population in European countries were thought to be higher compared to African or Asian countries and this might be contributed to the increase of stress level. Furthermore, the later spread of the COVID-19 infection in the other countries put them in a position to properly prepare the health care facilities for the worst scenarios, in addition to the early lockdown procedures and applying for the public precautions. Moreover, our responses from the Asian countries were mostly from Saudi Arabia, Emirates, and other Gulf countries where 
health care systems are well established and well equipped. It was found that working in the remote areas and private sectors were factors associated with stress in our cohort OR is 2.15 (1.22-3.80, CI 95\%), which could be related to lack of PPEs, the experience, and the staff who are ready to manage the pandemic.

The responses to the questionnaire in the present study showed that the most important factor that triggered stress in more than $80 \%$ of all the respondents was the fear of infection, where 949 (58.5\%) reported that fear of getting infected with COVID-19 increased their stress level while 384 (23.67\%) reported that the fear of transmitting the infection to their families was the main cause of their stress. These findings are in consistence with the results of Cai et al. [30] who found that concerns for personal safety, concerns for their families, and concerns for patient mortality were the important stress-triggering factors in the medical staff.

The intense media coverage of that pandemic with rapid spread of information, compared to the previous pandemics like SARS outbreak in 2003, H1N1 influenza pandemic in 2009, or the MERS-CoV epidemic in 2013-2015, could be another triggering factor for stress in the HCWs as reported by $3 \%$ of the respondents as it might exacerbate perceptions of personal health, which clearly exacerbated public fear, panic, and stress.

Multiple regression analysis of the significantly correlated variables showed female gender OR 1.88 (1.43-2.45, CI 95\%) and ICUs above capacity OR 3.33 (2.12-5.70, CI 95\%) and well above capacity OR 4.98 (2.81-8.24, CI 95\%), in addition to working in remote areas (including the private sector) OR 2.15 (1.22-3.80, CI 95\%), were independent predictors of stress.

Our study had some limitations, it was an electronic survey, and although this method is useful for rapid collection of data and is likely to produce similar results to paper method [31], ours had a small size convenient sample being a multicenter, international study. Moreover, because of the cross-sectional design, we were unable to account for or comment on the potential changes in the stress level over time which could be changeable with the change in the pandemic nature from country to another over time. Moreover, with a cross-sectional study methodology, it is hard to specify which comes first, the dependent or independent variables, and thus it was difficult to view the risk factors for stress; however, we viewed it as predictors and factors associated with stress. Furthermore, the cross-sectional design carries the risk of acquiescence bias (the friendly responder) versus extreme responding (the angry responder) and response bias where HCWs with stress tend to respond to the survey, while those without stress may not respond, which will bias the results toward high stress. Psychological stress can accumulate over time and have an impact later in the pandemic, including posttraumatic stress disorder
(PTSD), which should be investigated in future studies and its risk factors should be addressed in long-term studies. Additionally, we have not asked about the psychiatric history of the HCWs which could affect their responses to the survey results. Although we have used an objective tool for assessment of stress level (the PSS-10), but we still have some subjective responses for some studied items such as the effectiveness of the psychological support provided by the health care facility. Furthermore, we have received poor responses from some countries to build up a good power for statistical analysis which might turn the results not fully representative for the whole world.

\section{Conclusion}

Stress level was rated to be moderate to severe among intensive care HCWs during this pandemic, and many factors associated with stress were identified emphasizing the importance of psychological support during that unprecedented pandemic and adjusting different ways for mitigating such stress.

Supplementary information The online version contains supplementary material available at https://doi.org/10.1007/s11845-021-02721-0.

\section{Declarations}

Research involving human and animal participants This article does not contain any studies with human participants or animals performed by any of the authors.

Consent to participate Informed consent was obtained from all individual participants included in the study.

Conflict of interest The authors declare no competing interests.

\section{References}

1. World Health Organization (2020) Director-General's remarks at the media briefing on 2019-nCoV. https://www.who.int/dg/speeches/ detail/who-director-general-s-remarks-at-the-media-briefing-on2019-ncov-on-11-february-2020 (Accessed on 12 Feb 2020)

2. Le Gall JR, Azoulay E, Embriaco N et al (2011) Burn out syndrome among critical care workers. Bull Acad Natl Med 195(2):389-397

3. Schaufeli WB, Keijsers GJ, Reis Miranda D (1995) Burnout, technology use, and ICU-performance. In: Sauter SL, Murphy LR (eds) Organizational risk factors for job stress. APA Books, Washington, pp 259-271

4. Embriaco N, Azoulay E, Barrau K et al (2007) a: High level of burnout in intensivists: $\mathrm{M}$ prevalence and associated factors. Am J Respir Crit Care Med 175(7):686-692

5. Cohen S, Kamarck T, Mermelstein R (1983) A global measure of perceived stress. J Health Soc Behav 24:385-396 
6. Baik SH, Fox RS, Mills SD et al (2019) Reliability and validity of the Perceived Stress Scale-10 in Hispanic americans with english or spanish language preference. J Health Psychol 24 (5): 628-639

7. Cohen S, Williamson G (1988) Perceived stress in a probability sample of the United States. In: Spacapam S, Oskamp S (eds) The Social Psychology of Health. Sage, Newbury Park, CA, pp 31-67

8. Chua SE, Cheung V, Cheung C et al (2004) Psychological effects of the SARS outbreak in Hong Kong on high-risk health care workers. Can J Psychiatry 49(6):391-393. PubMed PMID: 15283534

9. Naushad VA, Bierens JJ, Nishan KP (2019) A systematic review of the impact of disaster on the mental health of medical responders. Prehospital Disaster Med 34:632-643

10. Lu YC, Shu BC, Chang YY, Lung FW (2004) The mental health of hospital workers dealing with severe acute respiratory syndrome. Psychother Psychosom 75(6):370-375

11. Maunder R, Hunter J, Vincent L et al (2003) The immediate psychological and occupational impact of the 2003 SARS outbreak in a teaching hospital. Can Med Assoc J 168(10):1245-1251

12. Zhu N, Zhang D, Wang W et al (2020) A novel coronavirus from patients with pneumonia in China, 2019. New Engl J Med 382:727-733

13. Lai J, Ma S, Wang Y (2020) Factors associated with mental health outcomes among health care workers exposed to coronavirus disease 2019. JAMA network open 3

14. Cohen S, Janicki-Deverts D (2012) Who's stressed? Distributions of psychological stress in the United States in probability samples from 1983, 2006, and 20091. J Appl Soc Psychol 42:1320-1334. https://doi.org/10.1111/j.1559-1816.2012.00900

15. Remor E (2006) Psychometric properties of a European Spanish version of the Perceived Stress Scale (PSS). Span J Psychol 9(1):86-93 PubMed PMID: 16673626

16. Uhlenhuth EH, Paykel ES (1973) Symptom intensity and life events. Arch Gen Psychiatry 28(4):473-477. PubMed PMID: 4692144

17. Kendler KS, Thornton LM, Prescott CA (2001) Gender differences in the rates of exposure to stressful life events and sensitivity to their depressogenic effects. Am J Psychiatry 158(4)587-593. PubMed PMID: 11282693

18. Dalgard OS, Dowrick C, Lehtinnen V (2006) Negative life events, social support, and gender differences in depression. A multinational community survey with data from the ODIN study. Soc Psychiatry Psychiatr Epidemiol 41:444-451

19. Rossi R, Socci V, Pacitti F et al (2020) Mental health outcomes among frontline and second-line health care workers during the coronavirus disease 2019 (COVID-19) pandemic in Italy. JAMA Netw Open 3(5):e2010185. https://doi.org/10.1001/jamanetworkopen.2020.10185

20. Li L, Cheng S, Gu J (2003) SARS infection among health care workers in Beijing. China JAMA 290(20):2662-2663. https://doi. org/10.1001/jama.290.20.2662

21. Shih FJ, Gau ML, Kao CC et al (2007) Dying and caring on the edge: Taiwan's surviving nurses' reflections on taking care of patients with severe acute respiratory syndrome. Appl Nurs Res 20(4):171-180. https://doi.org/10.1016/j.apnr.2006.08.007

22. Wong TW, Yau JK, Chan CL et al (2005) The psychological impact of severe acute respiratory syndrome outbreak on healthcare workers in emergency departments and how they cope. Eur J Emerg Med 12(1):13-18. https://doi.org/10.1097/00063110200502000-00005

23. Chan S (2003) Nurses fighting against severe acute respiratory syndrome (SARS) in Hong Kong. J Nurs Scholarsh 35(3):209. https://doi.org/10.1111/j.1547-5069.2003.00209.x

24. Zeng HM (2003) Fighting the SARS epidemic in Taiwan: a nursing perspective. J Nurs Adm 33(11):565-567. https://doi.org/10. 1097/00005110-200311000-00005

25. Mok E, Chung BP, Chung JW, Wong TK (2005) An exploratory study of nurses suffering from severe acute respiratory syndrome (SARS). Int J Nurs Pract 11(4):150-160. https://doi.org/10.1111/j. 1440-172X.2005.00520.x

26. Tyssen R, Vaglum P (2002) Mental health problems among young doctors: an updated review of prospective studies. Harv Rev Psychiatry 10:154-165

27. Loretto W, Popham F, Platt S et al (2005) Assessing psychological well-being: a holistic investigation of NHS employees. Int Rev Psychiatry 17:329-336

28. Shanafelt TD, Boone S, Tan L et al (2012) Burnout and satisfaction with work-life balance among US physicians relative to the general US population. Arch Intern Med 172:1377-1385

29. Shanafelt TD, Sloan JA, Habermann TM (2003) The well-being of physicians. Am J Med 114:513-519

30. Cai H, Tu B, Ma J et al (2020) Psychological impact and coping strategies of frontline medical staff in Hunan between January and, March 2020 during the outbreak of coronavirus disease 2019 (COVID19) in Hubei China. Med Sci Monit 26

31. Murray D, Fisher D (2002) The internet: a virtually untapped tool for research J Tech Human Serv 19:5-18

Publisher's Note Springer Nature remains neutral with regard to jurisdictional claims in published maps and institutional affiliations. 